\title{
La dimensión mediterránea de la proyección exterior de Cataluña: el Arco Latino
}

\author{
Caterina García Segura \\ Profesora Titular. Universitat Dompeu Fabra. Barceiona. Spain \\ Investigadora del Centre d'Estudis sabre la Pau i el Desarmament \\ Universitat Autònoma de Barcelona. 08193 Bellaterra (Barcelona). Spain
}

\section{Resumen}

La regín mediterránea constituye una de las áreas de la actividad exterior de Cataluña que mayor protagonismo está adquiriendo en los últimos tiempos.

La proyección exterior catalana, en su dimensión mediterránea, se caracteriza por perseguir, principalmente, objetivos de carácter económico. Por ello se articula alrededor del eje europeo-mediterráneo, el Arco Latino. Sin embargo, no pierde de vista la globalidad de la región ni la oportunidad y la necesidad de estrechar los vínculos con los países de la costa sur del Mediterráneo.

Palabras clave: proyección exterior subestatal, Cataluña, Meditertáneo, Arco Latino.

Abstract. The Mediterranean dimension of the external activity of Catalonia: the Latin Arch

The Mediterranean region is one of the areas which are taking more and more importance in the context of the external activity of Catalonia. The goals of the Catalonian external activity in its Mediterranean dimension are, mainly, of an economic character. That is why, this activity is articulated around the European-Mediteranean axis, the Latin Arch. However, it does not mean that Catalonia's external activity forgets the idea of globality of the region and, consequently, the opportunicy and necessity of enforcing the relations with the South Mediterranean countries.

Key words: non-state external activity, Catalonia, Mediterranean, Latin Arch.

\section{Sumario}

Los ejes prioritarios de la acruación exterior catalana: la vertiente mediterránea de Furopa

La concepción dual del Mediterráneo

El concepto de Arco latino y su problemática: modelos y escenarios de futturo
Los proyectos mediterráneos de la actividad exterior catalana

Conclusión

Bibliografía 
El Mediterráneo es una de las regiones que, por sus vínculos históricos, geográficos, culturales y económicos, constituye uno de los escenarios naturales de actuación exterior de Cataluña. Pero, antes que nada, hay que señalar que el Mediterráneo es un espacio dinámico, complejo y múltiple. Además de un espacio (un mar, un litoral), es un conjunto de realidades mucho más dificiles de definir (un "arte de vivir", una civilización) (El Malki, 1991: 15). En él se da una cierta convergencia de estilos de vida (identidad mediterránea), a la vez que una gran divergencia de culturas y políticas (identidades nacionales). $Y$ así, mientras que ciertos aspectos, aunque difusos o confusos, e incluso contestados, prometen una cierta homogeneidad del espacio mediterráneo, éste es también un mar de diversidades y contrastes político-económicos, sociales, culturales y religiosos. Algunas de estas oposiciones, la división Norte/Sur en términos económicos o la división musulmana/cristiana en términos de civilización, y sus consecuencias son focos de inestabilidad e inseguridad regional que deben ser tenidos en cuenta al elaborar una estrategia de actuación exterior respecto al Mediterráneo.

Para Cataluña, el espacio mediterráneo en su dimensión europea (Arco Latino, ribera norte) es una región, en conjunto, atrasada con respecto al conjunto nórdico-europeo-comunitario pero con enormes potencialidades de desarrollo económico. Su fuerza, para alcanzar los niveles anhelados, pasa por una estrecha y planificada cooperación regional. Sin embargo, es consciente que no puede separar el futuro del Arco Latino del de la ribera sur. Aunque de marcado acento europeo, la dimensión mediterránea de la actuación exterior de Cataluña se perfila sobre la necesidad de asumir y gestionar esta realidad como norte del Mediterráneo y sur de Europa.

\section{Los ejes prioritarios de la actuación exterior catalana: la vertiente mediterránea de Europa}

En 1993, el Comisionado para Actuaciones Exteriores de la Generalitat definió tres ejes básicos de la proyección internacional catalana. Estos eran, por orden de prioridad, Europa, Estados Unidos-Japón y América Latina'. De entre ellos, sin duda alguna Europa ha sido y es, mayoritariamente, el destino de sus actuaciones exteriores, tanto a nivel de definición de objetivos como a nivel de realizaciones prácticas. Un ejemplo significativo, en la medida que la actuación presidencial marca las pautas de la proyección exterior de la Generalitat en general, es el número de viajes del presidente de la Generalitat que han tenido como destino algún país europeo: 161 sobre un total de $176^{2}$. De estos 161,136 correspondieron a paises de la Europa comunitaria.

1. "Compareixença del Sr. Joan Vallvé i Ribera, comissionat per a Actuacions Exteriors, perquè informi dels projectes de la seva competèncian. Diari de Sessions del Parlament de Catalunya sèrie C, núm. 84, 28 d'abril de 1993, p. 2006.

2. Se han anatizado los viajes realizados por el presidente Pujol tentre enero de 1981 y julio de 1993. Sóto han sido contabilizados como destinos separados los casos en que se visitaban patses diferentes. Aquéllos en que se visitaban dos ciudades de un tmismo pafs han sido 
La dimensión mediterránea de la proyección exterior catalana se inscribe dentro del eje europeo. Este, en la definición del Comisionado para Actuaciones Exteriores, es conceptualmente muy vasto. Europa es considerada en un sentido amplio: comprende los países de la Europa comunitaria, los países del Esre y, en base a su pertenencia a la cuenca mediterránea, los países del norte de África, especialmente los del Meditetráneo occidental (Marruecos, Algeria y Túnez) cuya dinámica está fuertemente ligada y tiene claras consecuencias (económicas, sociales, etc.) para sus vecinos europeos de la costa norte.

Como veremos a continuación, el Mediterráneo, marco de referencia para la actuación exterior de la Generalitat, es todavía un espacio básicamente. europeo aunque exista esta voluntad incipiente de ampliarlo al espacio norteafricano.

\section{La concepción dual del Mediterráneo}

La actuación exterior de la Generalitat con respecto al Mediterráneo refleja dos concepciones del área. La primera y más utilizada se refiere exclusivamente a la vertiente europea del Mare Nostrum. La segunda lo amplía a los países del Magreb. En una y otra el Mediterráneo oriental queda al margen del escenario de acción, aunque sea considerado uno de los focos de mayor inestabilidad regional. Veremos que esto es consecuente con el objetivo perseguido por la actuación exterior catalana: la obtención de la seguridad regional a través del crecimiento económico.

La primera concepción destaca los elementos comunes entre los países del sur de Europa en contraposición a los rasgos que definen y configuran el conjunto norte-europeo. La segunda pone de relieve la línea Norte-Sur del sistema internacional, considerada como paradigmática de la complejidad que caracteriza el orden inundial contemporáneo, en el espacio mediterráneo.

La necesidad de aprehender esta doble vertiente es la clave para hallar soluciones satisfactorias a los retos contemporáneos derivados de la realidad mediterránea: desafíos geopolíticos, económicos, demográficos, ecológicos y socioculturales. La seguridad --en todas sus dimensiones: estratégico-militar, económica, ecológica, social, etc.- de los países del sur de Europa debe considerar el escenario global y el curopeo, pero debe añadirles también el magrebí. En este sentido, podemos afirmar que la segunda concepción de la política mediterránea catalana asume los retos del Mediterráneo sur como puntos de referencia.

A pesar de hallar todos estos elementos en diferentes declaraciones políticas de miembros del Gobierno de la Generalitat, la política mediterránea de

contabilizados como un único destino. Toda la información al respecto ha sido suministrada por la Subdirección General de Medios de Comunicación. Datos recogidos en el estudio de García Segura (1993). 
la Generalitat, al igual que la europea ${ }^{3}$, está todavía en fase de formulación en lo que a esta dimensión Norte-Sur se refiere. Mientras que la cooperación euromediterránea es ya una realidad con importantes proyectos en funcionamiento y redes estables de contactos, la vinculación con los países de la orilla sur del Mediterráneo es aún incipiente. Antoine (1993: 271) denuncia esta falta de movilización europea respecto a la costa sur del Mediterráneo: es imposible hallar solución para toda una serie de temas planteados (protección medioambiental, desarrollo estable) sin tener en cuenta las realidades, necesidades y posibilidades de ambas riberas.

En este sentido Cataluńa, que define su aproximación mediterránea dentro del marco europeo comunitario, apuesta por integrar esa otra vertiente. Trabajar para la consecución del equilibrio de todo el Mediterráneo y para sut renacimiento como área de prosperidad es la tarea planteada como la aportación catalana a la construcción europea.

Dentro de esta lógica, el presidente Jordi Pujol (Pujol, 1990) afirma que en el Mediterráneo hay una zona que tiene para Cataluña un especial interés -la que engloba el norte de Italia, el sur de Francia y la costa mediterránea de España-, pero que Cataluña contempla la globalidad de la región y que será necesario, sino ahora muy pronto, incluir en el área emergente del Mediterráneo occidental su ribera sur.

También el Institut Català d'Estudis Mediterranis, centro adscrito al Departamento de la Presidencia, recoge esta idea: «Mientras que la Europa de los Doce ya ha adquirido conciencia del hecho que stu destino va ligado al de los pueblos de la Europa Central y Oriental, no ha establecido aún un vínculo tan íntimo con el Mediterráneo. [...] De todos modos, a medio plazo, es casi inevitable, si no deseable, que se establezca una sinergia entre estos dos mundos" (Jouvenel \& Roque, 1993: 261).

Aunque el Magreb no ha sido hasta ahora un destino prioritario de la acción exterior de la Generalitat, tampoco ha estado totalmente ausente. En 1991 el presidente Pujol viajó a Túnez. Fue el primer viaje de Jordi Pujol a un país africano y tuvo una triple vertiente: cultural, económica y política.

A nivel cultural, se celebraron unas jornadas catalanas cuyo objetivo era contribuir a la definición de la mediterraneidad como condición esencial compartida por los países del norte y del sur.

A nivel económico, el objetivo del viaje era promover el incremento de inversiones catalanas en Túnez y fomentar las relaciones comerciales bilaterales. Acompañaba al presidente de la Generalitat una misión comercial. Como resultado de los contactos establecidos, se firmaron alrededor de veinte acuerdos comerciales con empresas tunecinas.

A nivel políico, el viaje obedeció a tres objetivos. En primer lugar, sirvió para plantear la visión mediterránea de la Generalitat. En segundo lugar, para llamar

3. La Política Mediterránea Renovada de la Comunidad para el período 1992-1996 es una opción en esta línea. Es el primer acuerdo de alcance regional con una visión de conjunto del Mediterráneo. 
la atención sobte la importancia que el Magreb tiene para España en su conjunto, Cataluña en particular y Europa en general. En último lugar, fue utilizado para incitar al Gobierno español a asumir mayores compromisos de cara al desarrollo de los países del Mágreb ${ }^{4}$. En este último aspecto, Jordi Pujol afirmó, durante su visita a Túnez, que la Generalitat abría áreas desatendidas por la política exterior española ${ }^{5}$.

Respecto a la premura de contribuir al desarrollo magrebí, afirmó que ello aportaría también ventajas evidentes para España en concreto y para la Europa mediterránea en general. Para la primera, puesto que contribuiría a aumentar la competitividad de los productos españoles que podrían ser producidos con menor coste en Túnez, Marruzcos o Argelia. Para la ribera norte, las ventajas derivarían del hecho que el desarrollo del Magreb contribuiría a frenar la ola migratoria hacia Europa ${ }^{6}$. Pujol señaló también que, para que el fomento de las inversiones europeas en el Magreb se convierta en realidad, estos países deben Ilevar a cabo un importante esfuerzo democratizador y de modernización y liberalización económica.

La idea de comprometerse a fondo con el desarrollo económico del Magreb ha sido retomada por el president de la Generalitat en orras ocasiones. En 1993, el president Pujol, en el Fórum Internacional Bertelsmann sobre Europa celebrado en Bonn, solicitó a los páses europeos la articulación de mecanismos de desarroilo para el Magreb como factor básico para la seguridad en el Mediterráneo. Pujol afirmó que: Eutopa no puede olvidar que tiene amenazada su frontera sur e instó abiertamente a la CEE a invertir en el área ${ }^{7}$.

Con ocasión de la apertura oficial del simposio celebrado en octubre de 1993 en Barcelona sobre "Los futuros posibles de la Europa mediterránea", Pujol reafirmó su propuesta de hacer que la cooperación con el Magreb se convierta en uno de los ejes clave de la política exterior del Estado español. En esta ocasión lo expresó estableciendo un paralelismo Magreb-Sudeste-asiático: planteó la posibilidad de que España fuera el motor del desarrollo del Magreb del mismo modo que Japón lo fue en su día de los Cuatro Dragones asiáticos ${ }^{8}$.

4. Véase, La Vanguardia, 3 de noviembre de 1991.

5. Vease, Avui, 5 de noviembre de 1991. Estas declaraciones originaron una protesta de los soctalistas catalanes. El PSC calificó como "ctíticas fueta de lugar" las alusiones del President Pujol acerca de la falta de compromiso de la diplomacia española con el Magreb. Véase, El Perídico, 7 de noviembre de 1991.

Anteriormente, el entonces rninistro de Asuntos Exreriores, Sr. Fernández Ordóñez, ya había reivindicado el eje mediterráneo de la política exterior espafiola. En la presentacion madrileña del libro del ICEM, fruto del simposium sobre "Els moviments humans en la Mediterrània occidental" el ministro empezaba así su intervención: "Parece innecesario decir a estas alturas que la política exterior de España apoya en el Mediterráneo una de sus líneas de acción más importantes" y continuaba: "España no es un país meditertáneo que está en Europa. Es un pats europeo que está en el Mediterráneo. [...] España no puede entenderse sin el Mediterráneo y por eillo no puede desentenderse de él». Presentación reproducida en La Vanguardia, 6 de octubre de 1990.

6. Véase, El Pats, 4 de noviembre de: 1991.

7. Véase, El Paús, La Vanguardia, 25 de octubre de 1993.

8. Véase, El Pats, 11 de octubre de 1993. 
En definitiva, se puede afirmar que aun cuando la mayor parte de los contactos mediterráneos de la Administración autonómica se llevan a cabo con los países de la Europa meridional, la política mediterránea de la Generalitat considera imprescindible, en un contexto de interdependencia, dotarse de esa perspectiva global que incluya a los países del sur.

\section{El concepto de Arco Latino y su problemática: modelos y escenarios de futuro}

Centrándonos en la dimensión euromediterránea de la proyección exterior catalana, entraremos en la definición del Arco Latino como espacio potencial de cooperación y colaboración.

El concepto de Arco Latino se ha ido desarrollando en un marco en el que se percibe la necesidad de una concepción global del Mediterráneo, pero en el que esa preocupación todavía no se ha traducido en políticas concretas. Claro reflejo de la complejidad regional, dicho concepto no es utilizado homogéneamente. En general, podemos decir que el Arco Latino sería el espacio norte del Mediterráneo respecto al sur que estaría constituido por el Magreb y el Mediterráneo oriental. También, a nivel general, se puede afirmar que el Arco Latino es concebido como un polo de desarrollo de la Europa del sur que, por sus vínculos con la Europa del norte y sus contactos privilegiados con los vecinos del sur meditetráneo, podría actuar como correa de transmisión del crecimiento económico y del desarrollo científico y tecnológico.

A nivel de extensión geográfica existen tres concepciones principales que difieren en cuanto a la amplitud del Arco Latino.

En primer lugar, el Institut d'Estudis Mediterranis considera como tal el conjunto regional que empieza en Valencia, pasa por Cataluña, la costa mediterránea de Francia y llega hasta Roma (Jouvenel \& Roque, 1993).

En segundo lugat, para la Dirección General XVI de la CEE (IFRN/CEE, 1992: I) el Arco Latino es la fachada sur de Europa sobre el Mediterráneo occidental y se extiende desde Andalucía hasta el Latium, incluyendo las isias de la cuenca oeste (Baleares, Córcega y Cerdeña) a excepción de Sicilia y Malta. Este conjunto de trece regiones, versión ampliada de la primera concepción presentada, es también el utilizado por Gaudemar (1991).

Una tercera definición es la presentada por Juan (1991). Para este autor, el Arco Latino está formado por un "núcleo duro" constituido por las regiones de Valencia, Cataluña, Languedoc-Roussillon, Provence-Alpes-Côte d'Azur, Liguria y Toscana, y por otro conjunto regional situado entorno a ese núcleo central: Castilla la Mancha, Aragón, Midi-Pyrénées, Rhône-Alpes, Piemonte, Lombardia, Emilia-Romagna y Latium. Esta concepción excluye Andalucía, como la primera, $\mathrm{e}$ incluye regiones no ribereñas.

A nivel conceptual, las tres coinciden. El Arco Latino, este conjunto euromediterráneo más o menos extenso, se caracteriza, en primer lugar, por el hecho de que las regiones que lo conforman comparten con las regiones del sur dos características particulares que las diferencian de las regiones del norte: 
a) el crecimiento demográfico mayor que el comunitario y b) los activos fenómenos migratorios, temporales o definitivos (Juan, 1991). Refiriéndose a este hecho, Roque (1990: 25) afirrna que la cuenca mediterránea es en la actualidad, junto con el Caribe, la región del mundo donde los Aujos y los movimientos migratorios son más intensos.

En segundo lugar, se trata de un territorio de dinámicas contradictorias en el que coexisten diferentes velocidades de desartollo fuertemente espacializadas. Por ejemplo, a nivel de $\mathrm{PIB} / \mathrm{hab}$. pueden distinguirse tres grupos de regiones: a) aquéllas de $\mathrm{PIB} / \mathrm{hab}$. inferior en un $25 \%$ a la media comuritaria (Andalucía, Valencia, Córcega, Cerdeña, Murcia); b) aquéllas con un $\mathrm{PIB} / \mathrm{hab}$. inferior en un $15 \%$ a la media comunitaria (Cataluña y LanguedocRousillon), y c) aquéllas con un PIB/hab. similar o superior a la media comunitaria (Provence-Alpes-Côte d'Azur, Liguria, Toscana, Ombria, Latium y Baleares) ${ }^{10}$.

A nivel de mercado de trabajo, el Arco Latino se caracteriza por una tasa de actividad (en particular femenina) inferior a la media comunitaria y por una tasa de paro superior a dicha media. También a nivel educativo podrían establecerse diferencias, por ejemplo, entre las regiones francesas, italianas y Cataluña y, en menor medida, Baleares y Valencia y el resto de las regiones del Arco.

No obstante, junto a las estructuras divergentes también existen dinámicas de convergencia, por ejemplo: el turismo y la orientación hacia las nuevas tecnologías representan factores de acercamiento.

En tercer lugar, aunque representan un peso económico relativo inferior a su peso demográfico en el seno de la Comunidad Europea, éste no es menospreciable, ya que si se toma, por ejemplo, la tasa de valor añadido, ésta evoluciona hacia valores que oscilan entre el $5 \%$ y el $15 \%$ de la media comunitaria (Juan, 91: 903).

En cuarto lugar, las regiones del Arco Latino comparten los factores culturales que las homogeneízan más allá de los comportamientos y modos de vida propios de la llamada «latinidad» o «mediterraneidad».

\section{Los modelos de desarrollo del Arro Latino}

En base a las velocidades y dinámicas de desarrollo divergentes, la DG XVI de las CEE distingue nada menos que once modelos de desarrollo (recordemos que las regiones que lo integran, según la misma DG XVI, son trece).

9. La tasa de crecimiento demográfico entre 1981 y 1990 fue del 3,7\%, superiot a la media europea $(3,1 \%)$.

10. Datos aportados por un informe de la DG XVI de la CEE (IFRN/CEE, 1992: II). Más recientemente, el boletín estadístico de EUROSTAT ha hecho públicos otros datos según los cuales las CCAA presentan situaciones económicas más favorables. De las incluidas en el Arco, Baleares y Cacaluria han alcanzado por primera vez un PIB/hab. superior y equivalente, respectivamente, al promedio comunitario. Citado por El Pats, 25 de enero de 1994. 
En primer lugar, el modelo andaluz, que se caracteriza por una situación en la que «[...] se está saliendo del subdesarrollo estructural pero en el marco de una lógica nacional voluntarista orientada por una relación privilegiada con Madrid y el centro de Españan (IFRN/CEE, 1992: IV).

En segundo lugar, el modelo del pás valenciano, caracterizado por la desarticulación y pot su situación "[...] a medio camino entre desartollo y subdesarrollo, entre reestructuración dolorosa y dinamismo industrial, entre internacionalización y repliegue sobre sí mismo o sobre el confort de las ayudas nacionales y comunitarias, entre gestión medioambiental y degradación ecológica acelerada» (IFRN/CEE, 1992: IV). Según los análisis de la DG XVI Murcia pertenecería a la vez al modelo andaluz y al valenciano.

En tercer lugar, el modelo catalán, caraterizado por "[...] una Cataluña motriz, orientada desde hace tiempo hacia la Europa del norte. A pesar de ciertos desequilibrios internos y a problemas reales de reestructuración, se mantiene como la locomotora de la economía española y, como tal, susceptible de conducir a un desarrollo multi-orientado [...]" (IFRN/CEE, 1992: IV).

En cuarto lugar, la fachada meditertánea francesa, que, en su calidad de auténtica encrucijada entre España e Italia, sería el escenario sobre el que se juega el futuro del Arco Latino. Las regiones que se integran en este modelo son regiones cruzadas por importantes ejes de carreteras, ferrocariles y vías marítimas que canalizan enormes flujos de mercancías e incluso de migraciones. La DG XVI estima que "La cuestión central respecto a su futuro concierne a su capacidad de captar una parte o la totalidad de estos flujos para asentar durablemente su desarrollo, particularmente para conseguir un verdadero despegue económico todavía no consolidado" (IFRN/CEE, 1992: V).

En quinto lugar, aparece el corredor ligur, detetminado por su situación entre los flujos de la poderosa Italia del norte y, en menor medida, los de la Italia central. Sus principales dificultades derivan del hecho que "[...] la reestructuración de sus viejas industrias podría conducir a una dependencia creciente de sus poderosos vecinos». Pero su situación geogràfica le permitiría "[...] según las eleciones estratégicas operadas, jugar un papel decisivo en la constitución de un Arco Latino de desarrollo" (IFRN/CEE, 1992: V).

El sexto modelo, el toscano, que cuenta con las ventajas de apoyarse sobre una buena red urbana al tiempo que practica un autodesartollo basado en las pequeñas y medianas empresas diversificadas y dinámicas, combinado con la extraversión económica, turística y cultural (IFRN/CEE, 1992: V).

En séptimo lugar, Roma o el Latium es un modelo inestable debido a que su "(...) esplendor económico es aún regional”, está "[...] pertubado al sur por la influencia napolitana" y es "(...) poco seguro al norte a pesar del desarrollo reciente de zonas de actividades a lo largo de la autopista del Sol o de la vía ferroviaria Roma-Florencia» (IFRN/CEE, 1992: V).

En tiltimo lugar, las islas que, a pesar de unos rasgos comunes (la insularidad y la fuerte dependencia estacional del turismo), constituyen casos separados. Así, el futuro de las Baleares, que han basado su desarrollo económico en el sector turístico, depende actualmente de su capacidad para superar y hacer 
frente a la crisis estructural del sector. Cerdeña, que presenta un turismo de gama alta y unas PIME interesantes, debe ser capaz de absorber ciertas caren.cias estructurales que limitan su crecimiento. Córcega, siempre según las estimaciones de la DG XVI, es la región del Arco de futuro más incierto a causa del clima de inseguridad y las desinversiones consiguientes, el envejecimiento de la población, el turismo masivo y muy concentrado en una temporada y toda una serie de desequilibrios estructurales.

\section{Los escenarios de desarrollo del Arco Latino"}

En paralelo a los diferentes modelos de desarrollo presentes en el Arco Latino, se plantean tres escenarios diferentes de futuro: el escenario tendencial, el escenario de la integración y el escenario alternativo de una integración sur-oeste de Europa.

El primero seguiría la "tendencia natural» de atracción Norte-Sur, dentro del conjunto europeo. Parte de la base de una periferia fragmentada que se desarrollaría, dependiendo del norte europeo, sobre un modelo de intercambios Norte-Sur. El Arco Latino se organizaría básicamente sobre cuatro polos fuertes - Barcelona, Lyon, Milán y Florencia-, que basarían su crecimiento en sus relaciones con el norte europeo. La consolidación de estos polos urbanos implicaría un lento declive demográfico y económico del resto del territorio.

El segundo es definido como "un escenario voluntatista de acercamiento de las regiones del Arco Latino: (IFRN/CEE, 1992: VIII). Supondría una suavización de las disparidades y una reducción de las fracturas que separan las regiones del Arco. Para ello requeriría, en primer lugar, una organización más eficaz de los intercambios que debería traducirse en el desarrollo de infraestructuras de transporte y de comunicación; en segundo lugar, debería llevarse a cabo una transformación profunda de las culturas y mentalidades, en el sentido de intentar elevar el nivel educativo y profesional de las regiones más atrasadas; en tercer lugar, sería deseable una gestión seria del desarrollo a nivel ecológico. Este escenario es concebido como un proceso gradual y organizado en diversos focos de trabajo: la constitución del eje Barcelona-Génova, el reequilibrio del corredor ligur, la consolidación del polo toscano articulándolo sobre el Arco Latino, la reducción de la fractura valenciana, la articulación para Murcia y Andalucia de un mecanismo complementario para anclar su desarrollo, la reinserción de Roma en el Arco Latino y, finalmente, un mayor acercamiento de las islas al conjunto regional.

El tercer escenario va más allá del Arco Latino propiamente dicho, ya que implica a todo el conjunto europeo. Se trataría de un gran proyecto de reequilibrio de la Europa sur-occidental que combinaría la complementariedad del Atco Latino y del Arco Atlántico y sería concebido "no como un nuevo "centro" en Europa, sino como una nueva dimensión en el centro europeo, en una perspectiva que se cabe esperar más igualitaria y más abierta a una coo-

I1. Estos madelos son también presentados por la DG XIV de la CEE (IFRN/CEE, 1992). 
peración con los "Sures", especialmente con el norte de Africa" (IFRN/CEE, 1992: XII).

En definitiva, el Arco Larino es una región con importantes potencialidades de crecimiento que coexisten con considerables disparidades que pueden constituir un freno para el desarrollo. Es en este sentido que se puede afirmar que el Arco Latino es más una ambición que una realidad. Es esa ambición la que inspira el movimiento de acercamiento entre las regiones que lo integran y es en esa linea que se perfila la dimensión mediterránea de la proyección exterior catalana. La realización de un escenario u otro dependerá del conjunto de las voluntades y las decisiones políticas tomadas, tanto a nivel comunitario, como estatal, regional o local.

\section{Los proyectos mediterráneos de la actividad exterior catalana}

Más allá de su definición o delimitación conceptual, la Europa mediterránea se está constituyendo en un foco nuevo de desarrollo económico, en una nueva "polaridad competitiva", con el desplazamiento de los tradicionales polos del centro y del norte de Europa hacia el sur. A pesar de todas sus disparidades, esta región muestra en su conjunto una gran vitalidad. Si se coge el caso de las regiones espaniolas se puede observar que a finales de los ochenta han tenido tasas de crecimiento compatables a las de Japón. Cataluña, por ejemplo, es la región mediterránea que tiene un crecimiento más rápido, junto con Turquía y Egipto (Jouvenel \& Roque, 1993: 267).

El Gobierno de la Generalitat quiere contribuir a potenciar la acción compensadora del actual desequilibrio entre el norte y el sur de Europa, es decir, favorecer la realización del escenario de la integración voluntarista. El president Pujol (Pujol, 1990: 33) afirma que Cataluña, como país del sur, está muy interesado en ello, pero añade que la reducción de las distancias responde también al interés general de Europa.

La Europa meridional no sólo debe preocuparse por alcanzar a la Europa del norte, sino que debe contribuir activamente a la construcción eutopea. Es por ello que el president Pujol reclama paralelamente para la Europa mediterránea un gran protagonismo en el renacimiento del Mediterráneo y en la integración europea.

En este sentido, Cataluña ha intentado sacar el máximo provecho de su posición fronteriza entre la Europa del Norte y la Europa del Sur. Por una parte, mantiene vínculos con las regiones más desatrolladas de Europa, por ejemplo, trabajando en la cooperación regional a través del programa ya mencionado de los Cuatro motores ${ }^{12} \mathrm{y}$, por otra parte, potencia el desplazamiento hacia el sur, por ejemplo, con la constitución de la Euroregión - Cataluña, Languedoc-Roussillon y Midi-Pyrénées-o con la creación de la Ruta de las

12. Programa de cooperación multisecrorial en el que participan las regiones de Cataluńa, Baden-Württemberg, Rhône-Alpes y Lombardia. A ellas se les anade, en acasiones, un quinto motor no europeo, la región canadiense de Ontario. 
Altas Tecnologías de la Europa del Sur. Estos víncuilos constituyen en la actualidad una estructura estable de contactos que favorecen los intercambios regionales (políticos, culturales, económico-funcionales, etc.), contribuyen al desarrollo económico de las partes y propician la consolidación del Arco Latino como espacio de dinamismo y crecimiento.

Como puede desprenderse de lo anterior, la actuación exterior de la Generalitat a nivel euromediterráneo coincide con su política de dotar a las entidades políticas subestatales de mayor peso político y de contribuir activamente al proceso de construcción europea desde el nivel regional.

A continuación vamos a describir sumariamente algunos de los instrumentos de integración regional que materializan los planteamientos euromediterráneos de la proyección exterior de Cataluña ${ }^{13}$.

\section{La Ruta de las Altas Tecnologías de la Europa del Sur}

La Ruta de las Altas Tecnologías de la Europa del Sur es una red de cooperación interregional definida en sus estatutos como Agrupación Europea de Interés Económico (AEIE) ${ }^{14}$.

La AEIE fue creada en octubre de 1990 a fin de fomentar la colaboración entre sus miembros a nivel de investigación y formación tecnológica, transferencia de tecnologías nuevas y ordenamiento de tecnopolos, de cata a favorecer y desarrollar la actividad económica de sus miembros y mejorar sus resultados. Este objetivo general se concreta en cuatro objetivos puntuales: 1) favorecer la cooperación industrial y el partenariado de las PIME y las PIMI a nivel de transferencias tecnológicas; 2) propiciar la participación común relativa a los programas de investigación y desarrolio; 3) promover la creación de la infraestructura de los tecnopolos y de los servicios comunes, y 4) colaborar en la formación tecnológica superior.

\section{La Euroregión}

La Euroregión fue constituida por los presidentes de Cataluña, LanguedocRoussillon y Midi-Pyrénées en octubre de 1991. Es un marco de cooperación

13. No existe pretensión de exhaustividad en la descripción que sigue. Se es consciente de estar excluyendo otras iniciativas que al no haberse dotado de una cierta estructura o a no haber pasado de la etapa de proyecto son de difícil sistemarización.

14. Según el reglamento CEE $2137-85$ de 25 de julio de 1885 , la ley núm. $89-377$ del 13 de junio de 1989 y todos los textos subsecuentes, así como por sus estatutos. Está integrada por el IMPIVA (Instituto de la Pequeña y Mediana Industria de la Generalidad Valenciana), el CIDEM (Centre d'Informació i Desenvolupament Empresarial adscrito al Departamento de Industria de la Generalitat de Catalunya), la AXISA, S.A. (Socjété d'Amenagement, de Gestion et d'Innovation sur les Nouvelles Tecnologies de Communication), el Multipôle Technologiqute Regional Languedoc Roussillon, la Route des Hautes Technologies, el DITEL (Centro Ligure per la Diffusione della Technologia), el Centro Estero Camete Cormmercio Piemontesi y el Milano Ricerche (Centro per la Constituzione del "Milano Ricerche", Centro per l'Innovazione in Citta Studi). 
interregional y transfronteriza al que se pretenden asociar las colectividades territoriales fronterizas, las universidades, las cámaras de comercio, los organismos consulares, las organizaciones profesionales, etc. Su objetivo es configurar un espacio coherente de desarrollo económico, científico, social y cultural.

La Euroregión está dotada de un comité tripartito encargado de coordinar las acciones ejecutadas por las tres administraciones, preparar una programación anual de las operaciones que deben realizarse en el seno de la Euroregión y garantizar su seguimiento. Cuenta además con seis grupos de trabajo sectoriales.

Siss objetivos esenciales son dos: 1) desplegar políticas de colaboración basadas en las aspiraciones de sus habitantes y facilitar los intercambios entre los actores económicos y culturales de las tres regiones, y 2) reforzar el papel de la Euroregión en el Mercado Único Europeo para favorecer la integración europea y el equilibrio de Europia en beneficio de las regiones del sur.

Estos objetivos deben alcanzarse a través de tres tipos de actuaciones: a) proyectos de cooperación interregional y transfronteriza decididos y promovidos directamente por las tres regiones; b) proyectos elaborados conjuntamente y realizados en el marco de las políticas comunitarias, y c) proyectos convergentes y coordinados de acciones realizadas simultáneamente en cada uno de los Estados implicados.

La Euroregión, a pesar de la naturaleza mediterránea de dos de sus tres componentes, está mucho más centrada en su dimensión pirenaica, pero la hemos incluido aquí porque sus objetivos de desarrollo y potenciación de la Europa del sur coinciden parcialmente con las formulaciones mediterráneas. Por el contrario no incluimos la Comunidad de Trabajo de los Pirineos, de la que también forman parte Cataluña y Languedoc-Rousillon ${ }^{15}$, porque aunque el reequilibrio regional de la Europa comunitaria es citado entre sus objetivos, la frnalidad principal - atenuar los aspectos negativos de la cadena montañosa en tanto que barrera fisica - se articula alrededor del hecho pirenaico.

\section{Carta de la cuenca mediterránea}

Firmada en septiembre de 1992, la Carta es un protocolo de intenciones dirigido a reforzar la cooperación entre los países y las regiones del contorno mediterráneo. Este instrumento presenta la particularidad de incluir, junto con las regiones mediterráneas de la $\mathrm{UE}^{16}$, dos países de la orilla sur, Marruecos y Túnez. Por lo tanto, combina los dos tipos de motivaciones para la cooperación: la potencialidad de la complementaneidad socioeconómica de las regiones europeas y la necesidad de reforzar los vínculos existentes entre ambas orillas del Mediterráneo. El objetivo de las regiones signatarias es profundizar sus relaciones en los sectores de la cooperación económica, la investigación, la formación, los transportes y la cultura. Para ello se articula un comité técnico de

15. La integran, además, Andorra, Aquitania, Aragón, Euskadi, Midi-Pyrénées y Navarra.

16. Provence-Alpes-Côte d'Azur, Cataluña, Liguria, Piemonte y Languedoc-Roussillon. 
cooperación, encargado de establecer las propuestas concreras, y un comité de elegidos, encargado de supervisar los trabajos del primero. En el seno del Comité técnico de coordinación se establecen diversos grupos de trabajo.

\section{Conclusión}

Podemos afirmar que la actuación exterior de la Generalitat en el área mediterránea se alinea perfectamente con su dinámica europeista, puesto que la articulación del Arco Latino se inserta en la lógica de consolidación de la integración europea. En primer lugar, porque el desartollo económico que persigue el Arco Latino tiene como finalidad última impulsar el reequilibrio entre la Europa del norte y la del sur. En segundo lugar, por su contribución a la potenciación de la Europa de las tegiones. Y, en tercer lugar, porque crea una dinámica de integración regional que abre la iniciativa gubernamental a la participación privada.

Cataluña, dentro del conjunto del Arco Latino, tiene un protagonismo específico derivado, en primer lugar, de su situación de frontera entre el norte y el sur de Europa - Cataluña es una región dinámica y en crecimiento dentro del Arco Latino-, y en segundo lugar, del convencimiento político del gobierno de la Generalitat en el futuro de la Europa de las regiones y del «Sunbelt» euromediterráneo.

La actuación exterior mediterránea de la Generalitat tiene una orientación y unos objetivos fundamentaimente económicos que, sin embargo, conjuga con objetivos políticos indirectos, como el fortalecimiento y la consolidación de la Europa de las Regiones.

Por otra parte, si bien la actuación exterior catalana respecto al Mediterráneo está concentrada en potenciar la cooperación entre las regiones europeas del Arco Latino, no ignora la importancia del papel desempeñado por los países del Magreb en la seguridad regional. El tema de la seguridad regional es abordado desde una perspectiva principalmente económica, pero sin olvidar los impactos políticos, sociales y culturales de los desequilibrios económicos.

Cabe destacar, en este sentido, su contribución al planteamiento de la urgencia de ampliación conceptual-funcional del Mediterráneo en la línea de incluir a los países magrebies. Supone asumir y aprehender uno de los imperativos de la realidad contemporánea. En este caso las identidades no son tan importantes como motores de la cooperación: Cataluña se define primero por su pertenencia a Europa; dentro de ésta, aparece en segundo lugar su vertiente mediterránea. Lo importante en este caso son las divergencias y los retos que de ellas emergen: crecimiento demográfico, dificultades económicas, fundamentalismo islámico, inestabilidad política y su traducción en flujos migratorios, contaminación medioambiental, aumento de la inseguridad regional, etc.

De la actuación exterior catalana en el ámbito mediterráneo se puede presumir que las opciones políticas van orientadas, a corto y medio plazo, hacia el escenario voluntarista de integración, pero sin perder de vista el escenario alter- 
nativo de integración global que, aunque se perfila como un escenario a largo plazo, tiene en cuenta el conjunto europeo y nuestro Sur más cercano, los países del Magreb.

\section{Bibliografia}

ANTOINE, Serge (1993). «18 pays riverains dans un même bateau? Le souci de l'environnement et du développement durable». La Méditérranée assassinée. Peuples Méditerrantens, núm. 62-63, p. 255-277.

Challiand, Gérand; MinCes, Juliette (1993). Etat de crise. Vers les nouveaux équilibres mondiaux. París: Seuil.

GAUDEMAR, J-P, DE (1991), «Problemàtica general i escenaris possibles de l'arc mediterrani", Revista Econòmica de Catalunya, núm. 18, septiembre-diciembre, p. 61. 70.

GaRCiA Segura, Caterina (1993). Balanç d'una decada de projeccio exterior de Catalunya. Parcialmente inédito.

El MALKI, Habib (1991). La Méditerranée en question. Conflits et interdépendances. Casablanca/Paris: Fondation du Roi Abdul-Aziz/Editions du CNRS.

IFRN/CEE. DG XVI (1992). Evolution prospective des régions de la Mediterranée Ouest. Rapport final provisoire. Bruselas: CEE.

JOUVENEL, H. DE; ROQUE, M.A. (directores) (1993). Catalunya a l'boritzb 2000. Prospectiva mediterrània. Barcelona: Institut d'Estudis Mediterranis.

JUAN, J-C. (1991). "Réalité de l'Arc Latin", en AAVV (1991). L'Avenir de l'espace méditerranéen. París: Editions Publisud, p. 896-912.

Nigoul, Claude (1991). "Quelques remarques sur les facteurs économiques et sociaux de l'insécurité en Méditertanéen, en EL MALXI. Op.cit., p. 123-147.

Pujol, Jordi (1990). "Catalunya i la Mediterrània", en Movimientos Humanos en el Mediterráneo occidental. Simposium intemacional Barcelona: Institut Català d'Estudis Mediterranis, p. 33-40.

RaVENEL, Bernard (1990). Méditerranée. Le Nord contre le Sud? París: Éditions L'Harmattan.

RoQue, M.A. (directora) (1990). Els Moviments Humans en el Mediterrani Occidental Barcelona: Institut Català d'Estudis Mediterranis. 